\title{
Efficacy and Safety of Lumen-Apposing Stents for Management of Pancreatic Fluid Collections in a Community Hospital Setting
}

\author{
Rajat Garg', Abdelkader Chaar ${ }^{2}$, Susan Szpunar ${ }^{3}$, Babu P. Mohan ${ }^{4}$ and Mohammed Barawi ${ }^{5}$ \\ ${ }^{1}$ Department of Internal Medicine, Cleveland Clinic Foundation, Cleveland, OH, ${ }^{2}$ Department of Internal Medicine, Ascension St. John \\ Hospital, Detroit, MI, ${ }^{3}$ Department of Biomedical Investigations and Research, Ascension St. John Hospital, Detroit, MI, ${ }^{4}$ Department of \\ Internal Medicine, University of Alabama, Tuscaloosa, AL, ${ }^{5}$ Division of Gastroenterology and Hepatology, Ascension St. John Hospital, \\ Detroit, MI, USA
}

Background/Aims: Endoscopic ultrasound-guided transmural drainage and necrosectomy employing lumen-apposing metal stent (LAMS) are used for treating pancreatic fluid collections (PFCs) with excellent results from academic centers. Herein, we report the efficacy and safety of LAMS in the treatment of PFCs at a community hospital.

Methods: We retrospectively reviewed the etiology of pancreatitis, type and size of PFCs, length of procedure, technical success, clinical success, adverse events, and stent removal. The primary outcome was the rate of clinical success, and secondary outcomes were technical success and adverse events.

Results: Twenty-seven patients with a mean age of $54.1 \pm 6.5$ years were included, $44 \%$ of which were men. The mean size of the PFCs was $9.7 \pm 5.0 \mathrm{~cm}$ (range, 3-21). The most common etiology of pancreatitis was alcohol (44\%) followed by idiopathic causes (30\%) and presence of gallstones (22\%). The diagnosis was pseudocyst in $44.4 \%(12 / 27)$ and walled off necrosis in $55.6 \%(15 / 27)$ of patients. There was $100 \%$ technical success without any complications. Clinical success was achieved in 22 of 27 patients (81.5\%) who underwent stent removal.

Conclusions: Our study is the first to report that endoscopic therapy of PFCs using LAMS is safe and effective even in a community hospital setting with limited resources and support compared to large academic centers. Clin Endosc 2020;53:480-486

Key Words: Endoscopic ultrasound; Lumen-apposing metal stent; Pancreatic fluid collection; Pancreatic pseudocyst; Self-expandable metallic stents

\section{INTRODUCTION}

Acute pancreatitis is a commonly encountered problem in a hospital setting. Patients with moderate-to-severe acute pancreatitis may develop pancreatic fluid collections (PFCs) with or without necrosis. The majority of PFCs spontaneously resolve without the need for any intervention; however, per-

Received: June 12, 2019 Revised: August 21, 2019

Accepted: August 21, 2019

Correspondence: Rajat Garg

Department of Internal Medicine, Cleveland Clinic Foundation, 9500 Euclid Avenue, Cleveland, $\mathrm{OH} 44195$, USA

Tel: +1-313-204-8093, Fax: +1-440-312-8588, E-mail: drgargrajat@gmail.com ORCID: https://orcid.org/0000-0003-1343-9939

cc This is an Open Access article distributed under the terms of the Creative Commons Attribution Non-Commercial License (http://creativecommons.org/ licenses/by-nc/3.0) which permits unrestricted non-commercial use, distribution, and reproduction in any medium, provided the original work is properly cited. sistently symptomatic PFCs require intervention. PFCs are classified using the Atlanta International Consensus Classification. ${ }^{1}$ PFCs that persists for four weeks after an acute episode are classified as pancreatic pseudocysts (PPs) containing homogenous fluid or as walled off necrosis (WON) containing both solid and liquid material. Lately, endoscopic ultrasound (EUS)-assisted drainage of PFCs has been shown to be better than surgical drainage in terms of complications, technical success, and cost effectiveness. ${ }^{2}$ EUS-guided transmural drainage and necrosectomy have become the standard treatment for patients with PFCs because of their safety and higher technical success rates than surgical and percutaneous methods. ${ }^{3,4}$ Lumen-apposing metal stents (LAMSs) are also successful in the management of PFCs and are utilized in a wide variety of procedures, including gallbladder drainage, gastrojejunostomy, and/or treatment of various gastrointestinal (GI) strictures. ${ }^{5-7}$ 
Most studies evaluating LAMS for PFCs have been conducted in large academic centers with limited data from community hospitals. The objective of this study was to assess the efficacy and safety of LAMS in the treatment of PFCs in a community hospital.

\section{MATERIALS AND METHODS}

This study was a single center retrospective chart review of adult patients (ages 18 years and older) with acute pancreatitis who underwent an EUS-guided transmural PFC drainage using LAMS (AXIOS; Boston Scientific, Marlborough, MA, USA) at our institution during a period of three years (January 2014-November 2017). Indications for the procedure were symptoms that included mainly abdominal pain (either from gastric outlet or biliary obstruction), or signs of infection (fever, elevated white cell count) in patients with previously known PFCs that have not been resolved on conservative management. Exclusion criteria included patients aged less than 18 years, coagulopathy (international normalized ratio $>1.5$ ), thrombocytopenia, or anatomy unsuitable for the procedure such as presence of blood vessels between the cyst and the tract wall or pseudocyst wall not in close proximity $(>1 \mathrm{~cm})$ to the EUS probe. Data were collected by two investigators (RG and $\mathrm{AC}$ ) after developing a standardized process to eliminate any subjective errors. We randomly checked the data of each investigator and any conflict was resolved by mutual consent. Our hospital's institutional review board approved the study.

\section{Technique}

All patients underwent EUS-guided LAMS placement using the AXIOS stent (Boston Scientific). All procedures were performed by an experienced interventional endoscopist (MB) under general anesthesia as per the hospital protocol. PFCs were evaluated and drained under linear array echoendoscope before stent placement. Initially, only non-cautery-enhanced LAMS (Cold AXIOS; Boston Scientific) were available; however, later subjects underwent cautery-enhanced LAMS; (Hot AXIOS; Boston Scientific) placement. Non-cautery-enhanced AXIOS stent placement included direct visualization of PFC, using $19 \mathrm{G}$ needle to puncture the collection, followed by passing the 0.035 inch guide wire through the needle and deployment of LAMS over guidewire under fluoroscopic and EUS guidance. Cautery-enhanced AXIOS stent placement involved puncturing the cyst wall using cautery tip under EUS guidance followed by deployment of LAMS immediately under EUS guidance. This process does not require the use of a guidewire and minimizes the time of exposure to radi- ation. During each procedure, a pancreatic fluid sample was aspirated and sent for analysis. The patients with positive fluid cultures from pancreatic aspirate were classified as infected pancreatic necrosis. The stent diameter $(10$ or $15 \mathrm{~mm})$ was left at the discretion of endoscopist. The decision to administer antibiotics was based on patients' comorbidities and periprocedural morbidity. We used the recommended follow-up after LAMS placement for stent removal of 10-14 days, the time needed for the tract to mature. All the patients had scheduled follow-up at two to four weeks based on interval cross-sectional imaging. Patients who demonstrated significant improvement were followed up earlier than patients with slow improvement on imaging. All LAMS were removed with grasping forceps.

We collected data on age, sex, date of stent placement, cause of pancreatitis, type of fluid collection, infection, size of stent $(\mathrm{mm} \times \mathrm{mm})$, location, technical success, clinical success, drainage site (transgastric or transduodenal), type of access (hot or cold AXIOS), proximal release of flange (direct or endoscopic with fluoroscopic visualization), length of procedure (min), sedation type, periprocedural antibiotics and major adverse events (bleeding, stent migration or perforation), number of required subsequent necrosectomies, date of stent removal, date of last/most recent imaging, complete resolution of PFC, and date of last follow-up. All adverse events were graded based on the American Society of Endoscopy lexicon guidelines. ${ }^{8}$ We did not collect data on coaxially or subsequently placed pigtail stents.

The primary outcome was the rate of clinical success (resolution of PFC, based on repeated radiological imaging) with technical success and adverse events constituted the secondary outcome. Patients were divided into two groups, those with PPs and those with WON. PFCs were classified after reviewing both the imaging (typically computed tomography [CT] scans) and EUS reports based on the revised Atlanta classification. Patients who had WON underwent direct endoscopic necrosectomies.

Technical success was defined as successful placement of the stent with subsequent observed drainage of the pancreatic fluid in the GI tract. Clinical success was defined as resolution of PFCs along with symptoms based on repeated imaging (EUS or CT scan). Partial resolution was defined as at least $75 \%$ decrease in size of PFCs on repeat imaging.

Major adverse events were periprocedural (within one day) bleeding, stent migration before removal or bowel perforation. All the adverse events were graded based on the American Society of Endoscopy lexicon guidelines. The follow-up period of adverse events was until the stent removal. 


\section{Statistical analysis}

Continuous variables were described using the mean and standard deviation or median and range. Categorical variables were reported as frequency distributions. Means were compared using Student's $t$-test. Mann-Whitney $U$-test was used for data that did not follow a normal distribution. The association between categorical variables was assessed using chi-squared analysis. Statistical analyses were performed using IBM SPSS Statistics for Windows, Version 25 (IBM Co., Armonk, NY, USA). A $p$-value of $\leq 0.05$ was considered statistically significant.

\section{RESULTS}

\section{Demographic and clinical characteristics}

We included 27 patients who underwent LAMS placement from January 2014 to November 2017. Twelve patients (44\%) had PPs while fifteen (56\%) had WON. The mean age of the patients was $54.1 \pm 16.5$ years; twelve $(44.4 \%)$ of which were male. The most common etiology of pancreatitis was alcohol (44.4\%) followed by idiopathic causes (29.6\%), presence of gallstones (22.2\%), and cystic fibrosis (3.7\%). There were no differences in etiologies between patients with PPs and WON. All the patients with PPs were drained as day care procedure whereas infected necrosis patients were treated as inpatient procedure as part of their acute care (Table 1).

\section{Pancreatic fluid collections characteristics}

The maximum diameter of PFCs ranged from 3 to $21 \mathrm{~cm}$ with a mean of $9.7 \pm 5.0 \mathrm{~cm}$. WONs were noted to be larger than PPs (11.3 \pm 5.1 vs. $7.8 \pm 4.1$ respectively, $p=0.07$ ).

The majority of PFCs were located in the pancreatic body, while the second most common site was the pancreatic tail. Only two patients had pancreatic head involvement (Table 1).

\section{Procedure details}

The mean procedure duration was $26 \pm 12.5$ minutes; the longest procedure took 60 minutes. All patients underwent general anesthesia. Almost all procedures (96.3\%) were performed using a transgastric approach while only one patient had a trasduodenal access. The hot AXIOS delivery system was used in 23 (85.2\%) patients while the remaining 4 (14.8\%) patients underwent treatment with the cold AXIOS delivery system. Two different stent sizes were used: $10 \times 10 \mathrm{~mm}$ and $15 \mathrm{~mm}$ wide $\times 10 \mathrm{~mm}$ long (44.4\% and 55.6\%, respectively).

Table 1. Patient and Pancreatic Fluid Collection Characteristics of Patients Who Underwent Pancreatic Fluid Collection Drainage

\begin{tabular}{|c|c|c|c|c|}
\hline & PFCs, $n=27$ & $\begin{array}{c}\text { Pseudocysts, } n=12 \\
\text { (Group 1) }\end{array}$ & $\begin{array}{c}\text { WON, } n=15 \\
(\text { Group 2) }\end{array}$ & $\begin{array}{c}p \text {-value } \\
\text { (Group } 1 \text { vs. Group 2) }\end{array}$ \\
\hline \multicolumn{5}{|l|}{ Sex, $n(\%)$} \\
\hline Male & & $6(50.0)$ & $6(40.0)$ & 0.60 \\
\hline Female & & $6(50.0)$ & $9(60.0)$ & \\
\hline Age, mean \pm SD (yr) & $54.1 \pm 16.5$ & $52.6 \pm 15.9$ & $55.3 \pm 17.4$ & 0.68 \\
\hline \multicolumn{5}{|l|}{ Pancreatitis etiology, $n(\%)$} \\
\hline Alcohol & $12(44.4)$ & $5(41.7)$ & $7(46.7)$ & \\
\hline Cystic fibrosis & $1(3.7)$ & $1(8.3)$ & $0(0.0)$ & \\
\hline Gallstones & $6(22.2)$ & $3(25.0)$ & $3(20.0)$ & \\
\hline Idiopathic & $8(29.6)$ & $3(25.0)$ & $5(33.3)$ & \\
\hline \multicolumn{5}{|l|}{ Infected PFC, $n(\%)$} \\
\hline No & $20(74.1)$ & $12(100)$ & $8(53.3)$ & \\
\hline Yes & $7(25.9)$ & $0(0.0)$ & $7(46.7)$ & \\
\hline \multicolumn{5}{|l|}{ Location, $n(\%)$} \\
\hline Body & $11(40.7)$ & $5(41.7)$ & $6(40.0)$ & \\
\hline Head & $1(3.7)$ & $1(8.3)$ & $0(0.0)$ & \\
\hline Tail & $2(7.4)$ & $2(16.7)$ & $0(0.0)$ & \\
\hline Body/head & $1(3.7)$ & $1(8.3)$ & $0(0.0)$ & \\
\hline Body/tail & $12(44.4)$ & $3(25.0)$ & $9(60.0)$ & \\
\hline Maximum PFC diameter, $\mathrm{mm} \pm \mathrm{SD}$ & $97.2 \pm 49.7$ & $77.8 \pm 41.5$ & $112.7 \pm 51.1$ & 0.07 \\
\hline
\end{tabular}

PFC, pancreatic fluid collection; SD, standard deviation; WON, walled off necrosis. 
Table 2. Procedural and Periprocedural Details of Patients Who Underwent Pancreatic Fluid Collection Drainage Using Lumen-Apposing Metal Stent

\begin{tabular}{|c|c|c|c|c|}
\hline & PFCs, $n=27$ & $\begin{array}{c}\text { Pseudocysts, } \\
n=12 \text { (Group 1) }\end{array}$ & $\begin{array}{c}\text { WON, } n=15 \\
\text { (Group 2) }\end{array}$ & $\begin{array}{c}p \text {-value } \\
\text { (Group } 1 \text { vs. Group 2) }\end{array}$ \\
\hline Duration of procedure, $\min \pm \mathrm{SD}$ & $26 \pm 12.5$ & $27 \pm 14.4$ & $25.2 \pm 11.1$ & 0.72 \\
\hline \multicolumn{5}{|l|}{ Drainage site } \\
\hline Transduodenal & $1(3.7)$ & $1(8.3)$ & $0(0.0)$ & \\
\hline Transgastric & $26(96.3)$ & $11(91.7)$ & $15(100.0)$ & \\
\hline \multicolumn{5}{|l|}{ Type of access } \\
\hline Cold AXIOS & $4(14.8)$ & $1(8.3)$ & $3(20)$ & 0.40 \\
\hline Hot AXIOS & $23(85.2)$ & $11(91.7)$ & $12(80)$ & \\
\hline \multicolumn{5}{|l|}{ Stent Size } \\
\hline $10 \times 10$ & $12(44.4)$ & $7(58.3)$ & $5(33.3)$ & 0.19 \\
\hline $15 \times 10$ & $15(55.6)$ & $5(41.7)$ & $10(66.7)$ & \\
\hline \multicolumn{5}{|l|}{ Proximal release of flanges } \\
\hline Direct visualization (hot) & $23(85.2)$ & $11(91.7)$ & $12(80.0)$ & 0.40 \\
\hline Endoscopic and fluoroscopic visualization (cold) & $4(14.8)$ & $1(8.3)$ & $3(20.0)$ & \\
\hline \multicolumn{5}{|l|}{ Sedation used } \\
\hline General anesthesia & $27(100)$ & $12(100)$ & $15(100)$ & \\
\hline \multicolumn{5}{|l|}{ Antibiotic used } \\
\hline Yes & $19(70.4)$ & $7(58.3)$ & $12(80)$ & 0.22 \\
\hline No & $8(29.6)$ & $5(41.7)$ & $3(20)$ & \\
\hline
\end{tabular}

PFC, pancreatic fluid collection; SD, standard deviation; WON, walled off necrosis.

Nineteen (70.4\%) patients received periprocedural antibiotics.

The data showed no difference between patients with PPs and WON in terms of procedure duration, drainage site, type of delivery system used, stent size, or use of antibiotics. No statistical test could be performed because of the small sample size (Table 2).

\section{Outcomes}

The median number of days from placement to removal was 31 days (range, 11-260 days). No major adverse events were reported. Technical success was achieved in all patients, whereas clinical success was observed in 22 of 27 patients (81.5\%). There were no occurrences of procedure-related bleeding, infection, obstruction, or clinically significant migration. There was one patient with asymptomatic stent migration on follow-up endoscopy. Patients with PPs and WONs had similar success rates, $83.3 \%$ and $80 \%$, respectively. None of the patients with PPs required any subsequent interventions, whereas almost half of those with WONs (47.7\%) underwent at least one subsequent necrosectomy. Out of 15 patients with WON, 7 had infected necrosis (cultures positive from pancreatic fluid aspirate) who required subsequent intervention. The number of subsequent interventions ranged from 1 to 5 with their clinical response guiding the need for re-intervention.
Median time interval after the procedure was 2 weeks.

Ten out of twelve (83.3\%) patients with PPs had complete resolution while the remaining two patients did not have data available. We only used data on technical success and immediate adverse events for patients who did not follow-up. In terms of WON, 10 of 15 cases (66.7\%) resolved completely, 2 (13.3\%) had partial resolution, and 3 (20\%) did not resolve. All our patients achieved symptom control despite residual PFCs on follow-up imaging, and none of the patients required percutaneous drain or surgical intervention (Table 3).

\section{DISCUSSION}

Our study demonstrates that LAMS is safe and effective for PFC drainage, even in a community hospital setting. Our findings are similar to those from large academic tertiary centers. ${ }^{9}$ LAMS has streamlined the endoscopic treatment of PFCs because of the ease of use and availability. The placement of LAMS was effective in $100 \%$ of the cases without any immediate post-procedural complications with $81.5 \%$ of patients achieving clinical success. Although there are multiple studies demonstrating the efficacy of this approach, our study is the first to evaluate it in a community hospital setting, 
Table 3. Follow-Up/Outcomes of Patients Who Underwent Pancreatic Fluid Collection Drainage

\begin{tabular}{|c|c|c|c|c|}
\hline & PFCs, $n=27$ & $\begin{array}{c}\text { Pseudocysts, } n=12 \\
\text { (Group 1) }\end{array}$ & $\begin{array}{c}\text { WON, } n=15 \\
\text { (Group 2) }\end{array}$ & $\begin{array}{c}p \text {-value } \\
\text { (Group } 1 \text { vs. Group 2) }\end{array}$ \\
\hline \multicolumn{5}{|l|}{ Technical success } \\
\hline Yes & $27(100)$ & $12(100)$ & $15(100)$ & \\
\hline \multicolumn{5}{|l|}{ Clinical success } \\
\hline Yes & $22(81.5)$ & $10(83.3)$ & $12(80.0)$ & \\
\hline No & $2(7.4)$ & $0(0.0)$ & $2(13.3)$ & \\
\hline Lost to follow-up & $2(7.4)$ & $2(16.7)$ & $0(0.0)$ & \\
\hline Being followed up currently & $1(3.7)$ & $0(0.0)$ & $1(6.7)$ & \\
\hline \multicolumn{5}{|l|}{ Major adverse events } \\
\hline No & $27(100)$ & $12(100)$ & $15(100)$ & \\
\hline \multicolumn{5}{|l|}{ Complete resolution of PFC } \\
\hline Yes & $20(74)$ & $10(83.3)$ & $10(66.7)$ & \\
\hline Resolving & $2(7.4)$ & $0(0.0)$ & $2(13.3)$ & \\
\hline No & $3(11.1)$ & $0(0.0)$ & $3(20.0)$ & \\
\hline Missing data & $2(7.4)$ & $2(16.7)$ & $0(0.0)$ & \\
\hline \multicolumn{5}{|c|}{ Number of required subsequent necrosectomies } \\
\hline 0 & $20(74.1)$ & $12(100.0)$ & $8(53.3)$ & \\
\hline$\geq 1$ & $7(25.9)$ & $0(0.0)$ & $7(47.7)$ & \\
\hline Time to removal, days, median [range $]^{\mathrm{a}}$ & $31[11-260]$ & $43.5[11-260]$ & $28.0[12-55]$ & 0.28 \\
\hline Removal to follow-up, median [range] $]^{\mathrm{a}}$ & $52[0-385]$ & $29.5[0-346]$ & $52[0-385]$ & 0.28 \\
\hline
\end{tabular}

PFC, pancreatic fluid collection; WON, walled off necrosis.

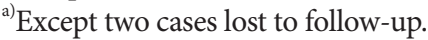

which demonstrates the wide applicability of this procedure.

The standard of care for the management of PFCs (including PPs and WON), adjacent to the gastric and duodenal lumen, involves internal drainage under EUS guidance. Endoscopic drainage of PFCs is highly preferred over alternative percutaneous or invasive surgical drainage, which is associated with high morbidity and mortality. Endoscopic drainage of PFCs is accomplished by creation of a controlled fistulous tract under EUS guidance, subsequently followed by the insertion of plastic biliary stents or fully covered self-expandable metal stents (FCSEMSs) for continued drainage. Transmural treatment of PFCs involves internal drainage of the collection into the bowel lumen. To perform endoscopic drainage, the fluid collection should be of reasonable size (generally $>3 \mathrm{~cm}$ ) and have a well-defined wall, with part of the wall of the fluid collection adherent to the GI tract lumen. Accessing the fluid collection is performed under EUS guidance, which allows for evaluation of the distance between the fluid collection and GI wall, detection of wall adherence, and identification of vessels that may be interposed between the two lumens. ${ }^{10}$

In the past, the procedure required guidewire insertion into the fluid collection, dilation to enlarge the transmural tract creating an endoscopic fistula, and placement of multiple plastic stents for drainage. ${ }^{10}$ The success of the procedure using plastic stents is affected by the type of PFCs and the size and number of plastic stents placed. Collections that are thick, necrotic, or infected may not adequately drain through plastic stents and may become secondarily infected after occlusion. ${ }^{11}$

More recently, a single step catheter has been used to accomplish drainage of these fluid collections under endosonographic guidance. FCSEMS with an electrocautery-enhanced delivery system have significantly larger lumens than those of plastic stents, and help create a lumen-to-lumen anastomosis that can keep the lumens in apposition to each other. ${ }^{12}$ Moreover, placement of LAMS also provides direct access to the cavity for further debridement with anchoring flanges preventing dislodgement.

The overall technical success rate exceeds $90 \%$ in the majority of published literature with complication rates around $10 \%-15 \%{ }^{9,13,14}$

The role of LAMS in the drainage of PFCs has been well established in multiple studies from large academic tertiary centers. ${ }^{9,11,14,15}$ Because the efficacy of LAMS in community hospital settings has not been studied, our goal was to as- 
sess our experience in our large urban community teaching hospital. Our study demonstrates that LAMS can be used effectively and safely in a community hospital, provided that an operator with sufficient expertise is available. We did not collect data on coaxially placed pigtail stents, and pigtail stent placement was left at the discretion of the endoscopist. Technical and clinical success rates were $100 \%$ and $81.5 \%$, respectively, without any immediate periprocedural complications. Interestingly, time to removal of LAMS appears to be shorter in patients with WON compared to those with PP. The likely reason of this finding is patients with WON required subsequent necrosectomies and closer follow-ups whereas patients with pseudocysts did not require subsequent interventions.

A recent study on 250 patients reported high rates of migration in patients undergoing LAMS placement for all indications. The rate of migration was $8.6 \%$ within the first three months for all indications, which increased to $48.5 \%$ at 12 months for PFCs compared to other indications that had a low (6.3\%) rate of migration. ${ }^{16}$ The study also found coaxially placed pigtail stents to be independently associated with an increased risk of migration. There were low rates of bleeding (5.4\%) related to stent placement, with most cases of bleeding occurring within one week of stent placement. Moreover, there were increased rates of bleeding with cold AXIOS (10) compared to hot AXIOS (3). Interestingly, three out of five patients with PFCs had delayed bleeding compared to other indications that had early bleeding. ${ }^{16}$ Our data are consistent with results of previous studies with a low rate of migration $(0 \%-19 \%) .^{17-19}$ The only migration in our study group was asymptomatic without any bleeding or obstruction.

LAMS with coaxially placed pigtail stents for PP drainage has been reported to be associated with low rates of infection compared to placement of LAMS alone ( $0 \%$ vs. $17 \%){ }^{20}$ Another study reported that placement of coaxial placed pigtail stent within LAMS was associated with a low risk of bleeding and infection as compared to placement of LAMS alone; however, most patients in the LAMS only group utilized the cold AXIOS technique which could have confounded their results. ${ }^{15}$ A recent study reported LAMS (92\%) to be more effective as compared to plastic stents (84\%). However, LAMS were obviously more expensive with an incremental cost effectiveness ratio favoring LAMS per additional patient successfully treated for treatment of WON. ${ }^{21}$

Our study has several limitations. First, it was a retrospective study with its inherent limitations. Second, there was no standardized follow-up for our patients. Third, endoscopies were performed by a single person, that might not reflect the true incidence of success in a large setting; however, this kind of expertise is not widely available and most patients are referred to tertiary centers from community hospitals. We also had variable rates of follow-up in our study with the longest follow-up of 385 days in WON and 346 days in PP group. The follow-up was dependent on the clinical visit and based on individual case. Many of our patients are still being followed up but due to small sample size and study completion, we could not factor ongoing follow-up. Furthermore, we did not separate patients with coaxially placed pigtail stents and subsequent placed pigtail stents for necrosectomies, which might have affected the outcomes.

Despite these limitations, our study has several strengths. Our study adds to the literature about the safety of the procedure and calls for wide acceptance of this minimally invasive procedure. We demonstrated for the first time that this procedure can be safely performed in community hospitals with outcomes as good as in academic centers.

In conclusion, our study reiterates the safety and efficacy of LAMS in treatment of PFCs even in community hospital setting and could widely provide the availability of expertise and institutional support. We also believe that advanced endoscopists at community hospitals can undergo training for this procedure by performing at least 25 procedures under the guidance of an experienced operator.

\section{Conflicts of Interest}

The authors have no financial conflicts of interest.

\section{REFERENCES}

1. Banks PA, Bollen TL, Dervenis C, et al. Classification of acute pancreatitis--2012: revision of the Atlanta classification and definitions by international consensus. Gut 2013;62:102-111.

2. Varadarajulu S, Bang JY, Sutton BS, Trevino JM, Christein JD, Wilcox $\mathrm{CM}$. Equal efficacy of endoscopic and surgical cystogastrostomy for pancreatic pseudocyst drainage in a randomized trial. Gastroenterology 2013;145:583-590.el.

3. Varadarajulu S, Christein JD, Tamhane A, Drelichman ER, Wilcox CM. Prospective randomized trial comparing EUS and EGD for transmural drainage of pancreatic pseudocysts (with videos). Gastrointest Endosc 2008;68:1102-1111.

4. Park DH, Lee SS, Moon SH, et al. Endoscopic ultrasound-guided versus conventional transmural drainage for pancreatic pseudocysts: a prospective randomized trial. Endoscopy 2009;41:842-848.

5. Mohan BP, Asokkumar R, Shakhatreh M, et al. Adverse events with lumen-apposing metal stents in endoscopic gallbladder drainage: a systematic review and meta-analysis. Endosc Ultrasound 2019;8:241-248.

6. Mohan BP, Shakhatreh M, Garg R, Ponnada S, Navaneethan U, Adler DG. Efficacy and safety of endoscopic ultrasound-guided choledochoduodenostomy: a systematic review and meta-analysis. J Clin Gastroenterol 2019;53:243-250.

7. Mohan BP, Chandan S, Garg R, et al. Lumen-apposing metal stents, fully covered self-expanding metal stents, and biodegradable stents in the management of benign of GI strictures: a systematic review and meta-analysis. J Clin Gastroenterol 2019;53:560-573.

8. Cotton PB, Eisen GM, Aabakken L, et al. A lexicon for endoscopic adverse events: report of an ASGE workshop. Gastrointest Endosc 
2010;71:446-454

9. Siddiqui AA, Adler DG, Nieto J, et al. EUS-guided drainage of peripancreatic fluid collections and necrosis by using a novel lumen-apposing stent: a large retrospective, multicenter U.S. experience (with videos). Gastrointest Endosc 2016;83:699-707.

10. Vila JJ, Carral D, Fernández-Urien I. Pancreatic pseudocyst drainage guided by endoscopic ultrasound. World J Gastrointest Endosc 2010;2:193-197.

11. Siddiqui AA, Dewitt JM, Strongin A, et al. Outcomes of EUS-guided drainage of debris-containing pancreatic pseudocysts by using combined endoprosthesis and a nasocystic drain. Gastrointest Endosc 2013;78:589-595.

12. Tyberg A, Karia K, Gabr M, et al. Management of pancreatic fluid collections: a comprehensive review of the literature. World J Gastroenterol 2016;22:2256-2270.

13. Alali A, Mosko J, May G, Teshima C. Endoscopic ultrasound-guided management of pancreatic fluid collections: update and review of the literature. Clin Endosc 2017;50:117-125.

14. Rinninella E, Kunda R, Dollhopf M, et al. EUS-guided drainage of pancreatic fluid collections using a novel lumen-apposing metal stent on an electrocautery-enhanced delivery system: a large retrospective study (with video). Gastrointest Endosc 2015;82:1039-1046.

15. Puga M, Consiglieri CF, Busquets J, et al. Safety of lumen-apposing stent with or without coaxial plastic stent for endoscopic ultrasound-guided drainage of pancreatic fluid collections: a retrospective study. Endosco- py 2018;50:1022-1026.

16. Garcia-Alonso FJ, Sanchez-Ocana R, Peñas-Herrero I, et al. Cumulative risks of stent migration and gastrointestinal bleeding in patients with lumen-apposing metal stents. Endoscopy 2018;50:386-395.

17. Chandran S, Efthymiou M, Kaffes A, et al. Management of pancreatic collections with a novel endoscopically placed fully covered self-expandable metal stent: a national experience (with videos). Gastrointest Endosc 2015;81:127-135.

18. Bang JY, Hasan M, Navaneethan U, Hawes R, Varadarajulu S. Lumen-apposing metal stents (LAMS) for pancreatic fluid collection (PFC) drainage: may not be business as usual. Gut 2017;66:2054-2056.

19. Siddiqui AA, Kowalski TE, Loren DE, et al. Fully covered self-expanding metal stents versus lumen-apposing fully covered self-expanding metal stent versus plastic stents for endoscopic drainage of pancreatic walled-off necrosis: clinical outcomes and success. Gastrointest Endosc 2017;85:758-765.

20. Aburajab M, Smith Z, Khan A, Dua K. Safety and efficacy of lumen-apposing metal stents with and without simultaneous double-pigtail plastic stents for draining pancreatic pseudocyst. Gastrointest Endosc 2018;87:1248-1255

21. Chen YI, Barkun AN, Adam V, et al. Cost-effectiveness analysis comparing lumen-apposing metal stents with plastic stents in the management of pancreatic walled-off necrosis. Gastrointest Endosc 2018;88:267276.e1. 